\title{
High-gradient behavior of a dipole-mode rf structure
}

\author{
B. Woolley, ${ }^{*}$ G. Burt๑, A. C. Dexter, R. Peacock, and W. L. Millar \\ Lancaster University, Bailrigg, Lancaster LA1 4YW, United Kingdom \\ N. Catalan Lasheras, A. Degiovanni, A. Grudiev, G. Mcmonagle, I. Syratchev, \\ W. Wuensch $\odot$, and E. Rodriguez Castro \\ CERN, 1211 Geneva 23, Switzerland \\ J. Giner Navarro \\ CERN, 1211 Geneva 23, Switzerland, and IFIC, 46980 Paterna, Valencia, Spain
}

(Received 27 September 2019; revised 25 July 2020; accepted 24 November 2020; published 17 December 2020)

\begin{abstract}
A normal-conducting, X-band traveling wave structure operating in the dipole mode has been systematically high-gradient tested to gain insight into the maximum possible gradients in these types of structure. Measured structure conditioning, breakdown behavior, and achieved surface fields are reported as well as a postmortem analysis of the breakdown position and a scanning electron microscope analysis of the high-field surfaces. The results of these measurements are then compared to high-gradient results from monopole-mode cavities. Scaled to a breakdown rate of $10^{-6}$, the cavities were found to operate at a peak electric field of $154 \mathrm{MV} / \mathrm{m}$ and a peak modified Poynting vector $S_{c}$ of $5.48 \mathrm{MW} / \mathrm{mm}^{2}$. The study provides important input for the further development of dipole-mode cavities for use in the Compact Linear Collider as a crab cavity and dipole-mode cavities for use in x-ray free-electron lasers as well as for studies of the fundamental processes in vacuum arcs. Of particular relevance are the unique field patterns in dipole cavities compared to monopole cavities, where the electric and magnetic fields peak in orthogonal planes, which allow the separation of the role of electric and magnetic fields in breakdown via postmortem damage observation. The azimuthal variation of breakdown crater density is measured and is fitted to sinusoidal functions. The best fit is a power law fit of exponent 6 . This is significant, as it shows how breakdown probability varies over a surface area with a varying electric field after conditioning to a given peak field.
\end{abstract}

DOI: $10.1103 /$ PhysRevAccelBeams.23.122002

\section{INTRODUCTION}

Dipole-mode rf structures play an important role in many particle accelerators [1], but at present little is known about the limits of their operation, with all designs currently using results scaled from accelerating structures. This paper experimentally studies the field limits in dipole (or deflecting) structures and analyzes the performance compared to accelerating structures to provide new insights into breakdown. Dipole-mode cavities in linacs are commonly used to provide longitudinal position-dependent, transverse momentum to a bunch. This can provide bunch longitudinal profile measurements or energy profiles when combined with a spectrometer [2]. Dipole-mode cavities

\footnotetext{
*Present address: CERN, 1211 Geneva 23, Switzerland.

Published by the American Physical Society under the terms of the Creative Commons Attribution 4.0 International license. Further distribution of this work must maintain attribution to the author(s) and the published article's title, journal citation, and DOI.
}

can also be used in emittance exchange systems [3], to provide ultrashort x-ray pulses in synchrotrons [4], or as kaon separators [5]. They can also be utilized as crab cavities which rotate a bunch prior to collision with another bunch to provide an effective head-on collision when the accelerator has a crossing angle [6]. In most cases, the dipole-mode cavities are placed in locations where space is at a premium, and, hence, increasing the deflecting gradient to allow shorter structures is important. In this paper, we make the consequentially important investigation of the maximum gradient for dipole-mode structures.

When metal structures in vacuum have a high electric field applied to them, a self-sustaining plasma discharge can be formed. The process is thought to start with excess field emission on emission sites, and this leads to a statistical phenomenon where the probability of a breakdown increases with the field level, and, hence, the mean time between breakdowns decreases with the field level. Many previous studies over the years have looked at how the breakdown rate in accelerating rf structures depends on peak electric fields, magnetic fields, and power flow in the 
structure [7-9]. While multiple dipole-mode cavities are in operation at many facilities, a systematic study of the breakdown rate has not been performed. Indeed, when operating cavities exhibit very few breakdowns [10], thereby suggesting higher gradients could be achieved, there is the potential option of using shorter structures with lower wakefields.

In this paper, we discuss the testing of a 24-cm-long $12 \mathrm{GHz}$ deflecting cavity. The cavity is a traveling wave structure with 12 cells operating in the $2 \pi / 3$ mode and is a prototype of a high-gradient deflector intended for use as a dipole mode in a future free-electron laser (FEL) and as a crab cavity for the Compact Linear Collider (CLIC) [11]. In both applications, a high gradient is preferred to minimize the structure length. For a dipole-mode cavity placed just before or after an FEL, a high transverse voltage is required due to the high beam energy and the short bunch length. This often requires a long deflector or in many cases multiple deflectors. A high gradient would allow the saving of space in this critical region of an FEL. For a crab cavity, the cavity is positioned in a region where the transverse deflections can provide the largest deflection for a given kick; however, this also makes the cavity very sensitive to wakefields which are proportional to the cavity length [12] — hence, shorter cavities are again preferred. A study of the breakdown rates of a dipole cavity versus input power would allow some benchmark to be used for the design of these structures to the maximum gradient.

In this paper, Sec. II describes the design of the prototype cavity, giving field distributions and locations for maxima. Section III describes the measurement setup and diagnostics employed at CERN's XBox-2 test stand for this study. The first stage of high-gradient tests is conditioning. This is a process whereby sites in the cavity with excessive field emission, that leads to breakdown, caused by impurities or surface defects such as field enhancement tips are burned away by slowly increasing the power. Conditioning of the dipole cavity is described in Sec. IV. Measurements after conditioning and at the highest gradients showing how breakdown correlates to various figures of merit are presented in Sec. V. Section VI gives a postmortem analysis on the sectioned cavity relating surface damage to local fields.

\section{DIPOLE CAVITY DESIGN AND MANUFACTURE}

For any optimization, it is normal to set goals on the peak surface fields, but, in this case, it was not clear what these limits should be based on previous experience [13]. Data do exist from dipole-mode cavities installed on machines, but, as there are so few breakdowns in these structures, this can set only lower limits. For the Linac Coherent Light Source (LCLS) deflector [10], the operating peak surface electric and magnetic fields are $115 \mathrm{MV} / \mathrm{m}$ and $405 \mathrm{kA} / \mathrm{m}$, respectively. This is roughly half the peak surface electric field in CLIC T24 accelerating structures [14] and almost the same peak surface magnetic field; hence, it is not clear if this is close to the limit or far from it. In addition to this, recent studies [7] have suggested alternative figures of merit for estimating breakdown such as power flow over circumference or a modified Poynting vector $\left(S_{c}\right)$.

However, judging the role of each field quantity is difficult in accelerating structures, because the peak electric fields, magnetic fields, and Poynting flux all occur at roughly the same point on a cavity's iris. Conversely, dipole cavities (normally operating in a hybrid $\mathrm{TM}_{11} / \mathrm{TE}_{11}$ mode, referred to as the $\mathrm{HE}_{11}$ mode) have peak fields in different locations on the iris, and a horizontally polarized cavity has its peak electric field in the horizontal plane, its peak magnetic field in the vertical plane, and its peak Poynting vector at an angle of $45^{\circ}$ with respect to the horizontal and vertical planes, as shown in Fig. 1. This fact allows the role of each field component to be inferred from the damage location on postmortem inspection after a high-gradient test. The results for this test, thus, can to contribute to future development of high-gradient limits.

The dipole-mode test cavity, the CLIC crab cavity prototype, has a racetrack cross section so that the two polarizations of the $\mathrm{HE}_{11}$ mode are at different frequencies. The major axis diameter is $29.16 \mathrm{~mm}$, and the minor is $24.13 \mathrm{~mm}$, giving a $1 \mathrm{GHz}$ frequency difference between the two polarizations [11]. A phase advance of $120^{\circ}$, with an aperture diameter of $10 \mathrm{~mm}$ and a disk thickness of $2 \mathrm{~mm}$, was chosen as a compromise between group velocity
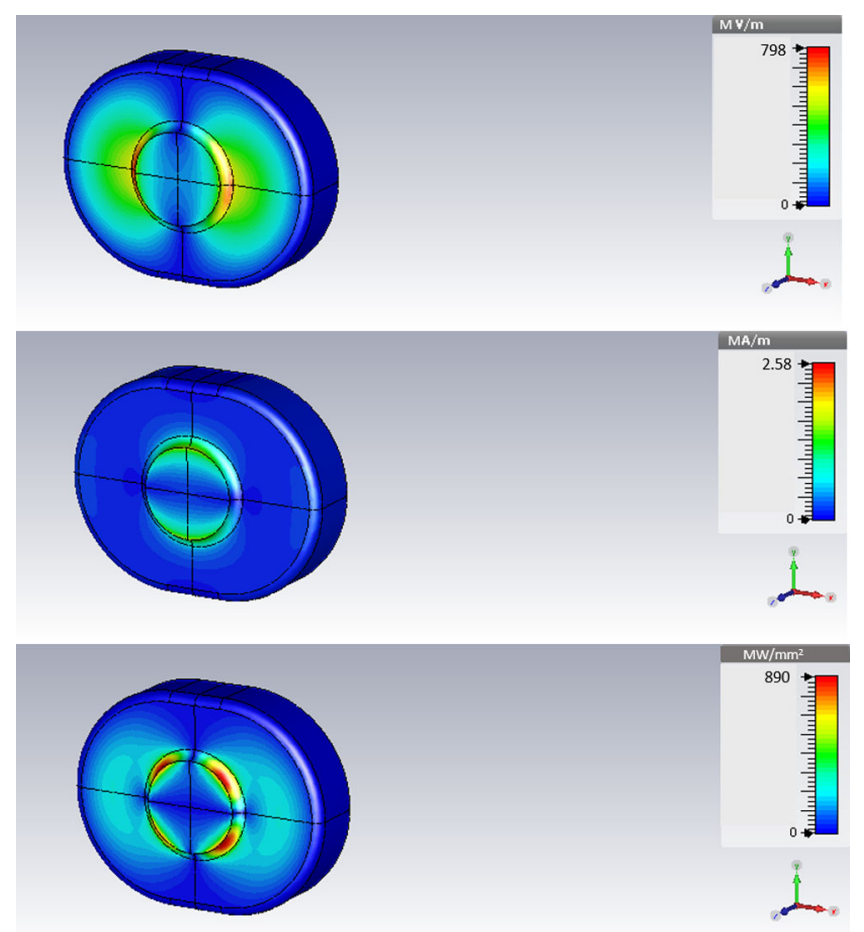

FIG. 1. Surface electric (top), magnetic (middle), and Poynting flux (bottom) for the dipole-mode cavity with $1 \mathrm{~J}$ stored energy per cell. 


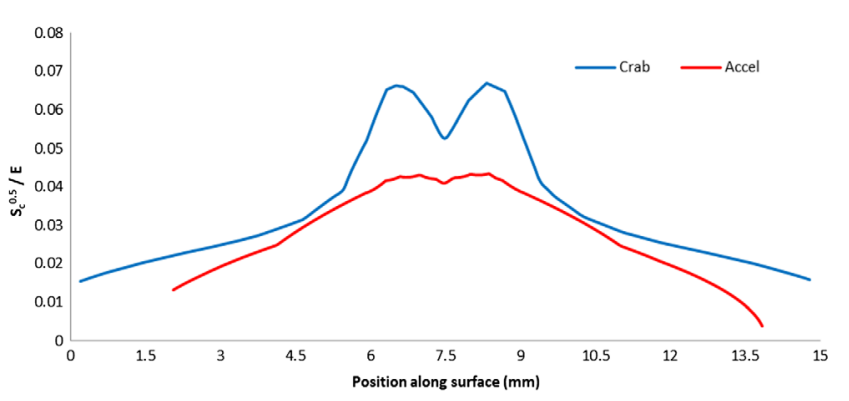

FIG. 2. Comparison of $\sqrt{S_{c}}$ divided by the cavity gradient along the aperture and wall surface for a dipole-mode "crab" cavity and an accelerating cavity with the same aperture radius and thickness where the center of the iris is at $7.5 \mathrm{~mm}$ in both cases.

and shunt impedance. Comparing $12 \mathrm{GHz}$ accelerating and the dipole-mode test cavity, one finds that, for the same accelerating or deflecting gradient, the dipole-mode cavity will have a $40 \%$ higher peak electric field and a $130 \%$ larger peak magnetic field. This results in a modified Poynting vector $S_{c}$ that is 2.4 times higher, as shown in Fig. 2. Comparing the two structures at the same peak surface electric field, the peak magnetic field is $60 \%$ larger, and it has an $S_{c}$ which is $18 \%$ larger.

The 12-cell prototype dipole-mode cavity was machined at VDL [15], in Netherlands. The surface roughness specification was $\mathrm{Ra}=24 \mathrm{~nm}$, which was achieved on all cells. This structure was diffusion bonded rather than brazed in order to most directly compare performance to high-gradient accelerating cavities tested as part of the CLIC project at CERN. The bonding was performed by Bodycote [16], in France. The final structure is shown in Fig. 3.

The 12-cell prototype dipole-mode cavity was tuned [17], proceeding cell by cell from the output to the input. At each step, a bead-pull measurement was performed to calculate the electric field pattern (of $E_{y}$ ). Using the measured field, the cell under consideration was tuned with

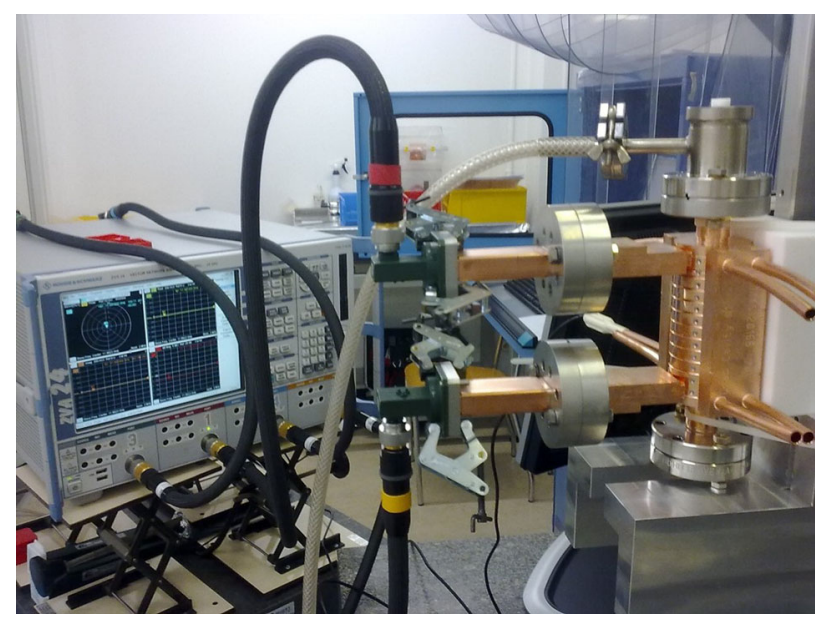

FIG. 3. Cavity 2, manufactured by VDL.
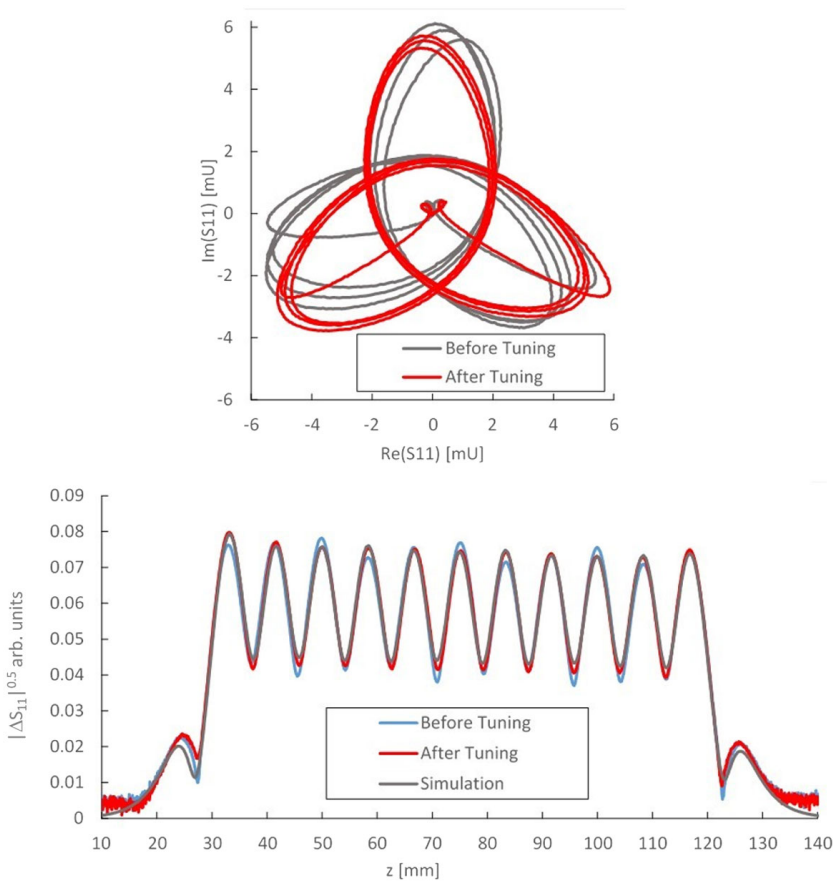

FIG. 4. Bead-pull measurement results before (blue lines) and after (red lines) tuning compared to simulations (gray lines).

wall deflections forced with a slide hammer attached to a brazed tuning pin (allowing both a frequency increase and decrease) while observing $\Delta S_{11}$. Cell by cell, the bead-pull measurement was repeated, and the overall effect of a cell's tuning operation was evaluated. Bead-pull measurements and the tuning of each cell were repeated until the electric field pattern was close to the final specification. Occasionally, a cell that had already been tuned had to be retuned. After 26 steps, the phase advance per cell of all ten regular cells was $120^{\circ} \pm 0.1^{\circ}$. The final bead-pull measurement is shown in Fig. 4.

\section{MEASUREMENT SETUP AND DIAGNOSTICS}

The cavity performance was tested with high-power rf at XBox-2, which is one of CERN's X-band test stands [18]. Cavity installation and diagnostics at XBox-2 are shown in Fig. 5. The XBox-2 test stand is one of three high-power $\mathrm{X}$-band test stations at CERN. XBox-2 was originally powered by a 50 MW CPI XL5 klystron, until this unit developed a fault during testing and was replaced with a CPI VKX-8311A tube. The test stand utilizes a pulse compressor [19] which was bypassed for part of the testing by detuning the pulse compressor cavity. When the pulse compressor operates, a small fraction of the peak power is delivered before and after the main pulse as given in Ref. [19]; potentially, this may have affected the breakdown performance of the cavity, and there is evidence for this in the data.

Directional couplers are used to sample incident, reflected, and transmitted power signals. Sampled signals 


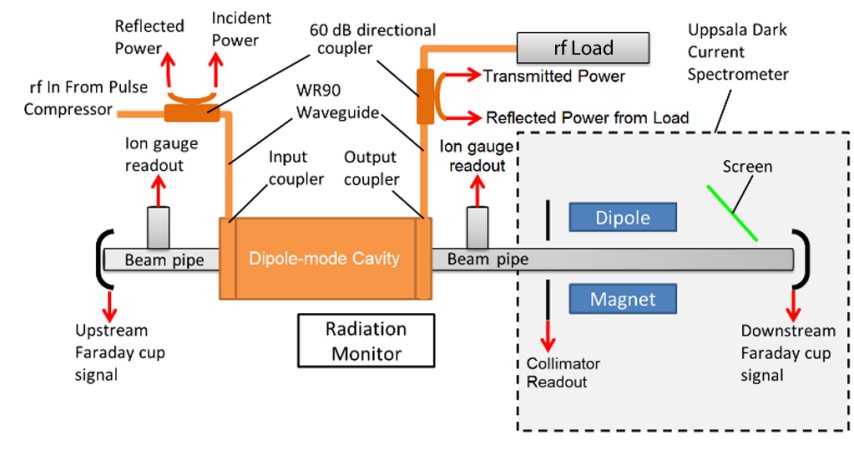

FIG. 5. Cavity testing setup at XBox-2.

were divided for separate power measurements and downconverted to $400 \mathrm{MHz}$ for digital sampling. The upstream beam pipe is connected to a Faraday cup, while the downstream beam pipe has a collimator and a dark current monitor [20]. These instruments measure the charge emitted due to field emission or breakdown. Both beam pipes also have ion gauges to measure the vacuum pressure in the cavity.

A breakdown is detected when the reflected power signal exceeds a threshold limit. The data from all couplers are then analyzed to indicate where the breakdown occurred. The first analysis step is to determine whether the event occurred within the structure or elsewhere in the waveguide network. This is done by looking at which directional couplers reached the threshold reflected power, referred to here as the breakdown flags, and which are recorded for every interlock event. This allows the location to be known to within the length of the waveguide between two directional couplers. For example, if there is a breakdown in the structure, there will be a threshold breach in the "reflected from structure" channel (PSR) and possibly at the "reflected to klystron channel" (PKR). However, there will be no threshold breach on the "load reflected channel" (PER). It is possible that the breakdown could occur in one of the short lengths of waveguide connecting the structure to the directional couplers; however, an assumption has been made that the probability of an event occurring in the structure is much higher than that for a waveguide, because the surface fields are generally an order of magnitude lower in the waveguide.

When a breakdown occurs, it acts as a short circuit, reflecting the power back to the input and causing the transmitted signal to fall. The breakdown location can, therefore, be distinguished using the time delay between the rising and falling edges of the reflected and transmitted signals reaching the input and output directional couplers, respectively [21]. Since the dipole-mode cavity is a constant impedance structure, the group velocity is constant throughout the structure, and, hence, the time delay is easily translated into a location. A second method of locating the breakdown location is to look at the delay between features in the incident and reflected signals, such as those created by reflections from the arc reaching the klystron [20].

\section{STRUCTURE CONDITIONING}

Before testing the structure at high power, it had to be conditioned by slowly increasing the rf power in steps after a number of pulses without breakdown, such that high fields could be sustained without causing irreparable damage to the structure. Conditioning and testing took several months. Conditioning was performed with increasing power levels and, hence, increasing surface electric fields. Power levels were adjusted and sometimes cut back for a period to limit the number of breakdowns based on a target breakdown rate (BDR). Conditioning is normally started with shorter pulse lengths to keep the initial BDR low which are later increased. The variation with surface electric field and pulse length has been documented; hence, scaling to a nominal pulse length and surface electric field provides an informative plot of the whole conditioning process. The purple trace in Fig. 6 provides this information for the dipole-mode cavity where the BDR has been scaled to a 200 ns pulse length and to a surface electric field of $200 \mathrm{MV} / \mathrm{m}$. Figure 6 also compares conditioning of the dipole-mode cavity to two tests of CLIC TD24R05 accelerating structures [18].

Initially, the flattop peak duration was set to $100 \mathrm{~ns}$; this reduces potential damage after an arc compared to the full operating pulse length pulse of $180 \mathrm{~ns}$. The power was initially ramped manually over a few minutes until the first breakdown event was observed. This occurred at a power level of 4.5 MW. The power was then reduced by $10 \%$ and control handed to an automatic conditioning algorithm. The conditioning algorithm controls power, so the breakdown rate is steady at a set point chosen in this instance as $5 \times 10^{-5}$ breakdowns per pulse (bpp). The power ramp is held when set target powers are achieved. The first target level was $10 \mathrm{MW}$, which is slightly below the operational

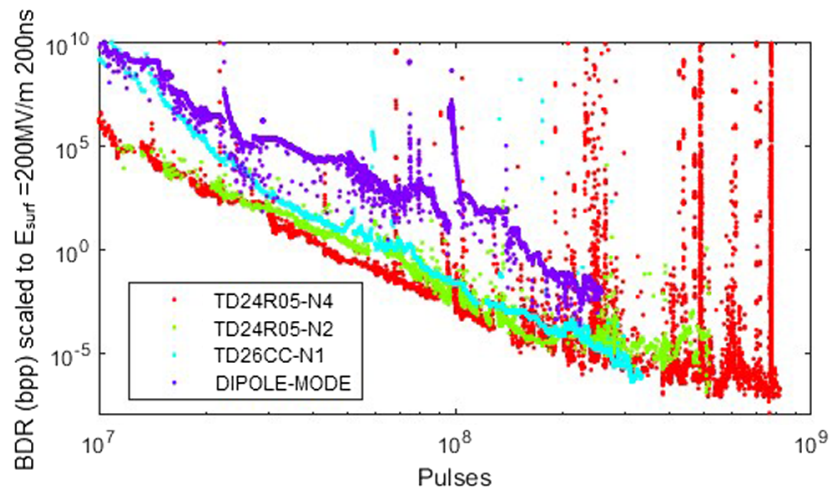

FIG. 6. Breakdown rate scaled to $200 \mathrm{MV} / \mathrm{m}$ at $200 \mathrm{~ns}$ for the whole conditioning history of the dipole-mode cavity versus high-gradient monopole structures tested at CERN. Accelerating structure data are from Ref. [14]. 
power, and the second target power level was set to 15.5 MW, which is $16 \%$ higher than the nominal operational power level. After another 7.5 million pulses, the first target was reached, while keeping the BDR below $6 \times 10^{-5} \mathrm{bpp}$. The flattop pulse duration was then increased to $200 \mathrm{~ns}$ and the power lowered to $11 \mathrm{MW}$. After just 3 million pulses, the second target power level of 15.5 MW was reached, and the structure was ready for a long run at nominal operating conditions. Note that Fig. 6 starts at 10 million pulses.

For the nominal operational run, the power level was set at a fixed value of 14.2 MW, which is slightly higher than the operational power (13.3 MW). During the first half of the run, the BDR fell in an exponential manner until it reached a BDR of $2 \times 10^{-6} \mathrm{bpp}$. This BDR is maintained for another 12 million pulses until the end of the run, showing that the dipole-mode cavity can run successfully at its nominal operating conditions. With further running at this power level, it is likely that the BDR would continue to decrease, albeit quite slowly.

After testing at slightly above the operational power, the cavity was pushed to its maximum gradient to find its ultimate operating point. The goal was to see if the structure could be further optimized to make it shorter and, hence, reduce the wakefields. The flattop pulse width was lowered back to $100 \mathrm{~ns}$ and the power level increased manually until the first breakdown occurred at 19 MW. The power was reduced back to $18.5 \mathrm{MW}$, and the conditioning algorithm was restarted with the same $5 \times 10^{-5}$ bpp set point. No ultimate power level goal was set, as it was unclear what power level the cavity could reach. The conditioning algorithm ramped the power up steadily until it reached $26 \mathrm{MW}$, when a cluster of breakdowns occurred after around 60 million pulses, and the power was sharply decreased by the algorithm. This cluster is seen in Fig. 6 as a spike. Note that this figure gives scaled BDR and not $\mathrm{BDR}$. At this point, to try and protect the structure from too many breakdowns, the BDR set point was lowered slightly from $5 \times 10^{-5}$ to $4 \times 10^{-5} \mathrm{bpp}$. After changing the set point, the power level recovered and reached $27 \mathrm{MW}$ before another cluster of breakdowns occurred, suggesting a hard limit had been reached. This spike can also be seen in Fig. 6 . After 72 million pulses, the power level was reduced to $25 \mathrm{MW}$ to see if the structure was stable at this power level. After a further 6 million pulses at $25 \mathrm{MW}$, the structure was running stably with a BDR of $1.5 \times 10^{-5} \mathrm{bpp}$. The flattop pulse duration was then increased to $200 \mathrm{~ns}$, and the power was set to $20.3 \mathrm{MW}$. At this power level, the integrated kick is $3.14 \mathrm{MV}$ compared to the baseline $2.55 \mathrm{MV}$. Over the next 15 million pulses, the BDR continued to fall to $(1.9 \pm 0.3) \times 10^{-6} \mathrm{bpp}$, suggesting that the structure was still conditioning.

After 92.5 million pulses, the XL5 klystron from SLAC was replaced with a new CPI VKX-8311A tube. To install the klystron, the waveguide vacuum had to be broken.
The replacement work took one week, over which the entire time the structure was exposed to the atmosphere. Because of this, on restarting, the power level and pulse width were reduced to $15 \mathrm{MW}$ and $50 \mathrm{~ns}$, respectively, as it was expected that some reconditioning of the structure would be required. The power was ramped manually to $20.3 \mathrm{MW}$ very quickly, retaining the $50 \mathrm{~ns}$ pulse width. After this, the flattop pulse duration was increased to $200 \mathrm{~ns}$, and the conditioning algorithm was reactivated with the target power set to the original 20.3 MW. The target was reached after 5 million pulses. Figure 6 shows a break in the purple trace when the klystron was replaced and a high breakdown rate during initial conditioning after breaking the vacuum.

Up until the aging XL5 klystron was replaced with the new VKX-8311A, a pulse compressor was needed to provide the required power for testing. With the new klystron being able to supply the full $50 \mathrm{MW}$, use of the pulse compressor was not necessary. To compare the performance of a structure that has a compressed input pulse to that without a compressed pulse, the pulse compressor was detuned. It might be anticipated that the breakdown rate could be higher for a compressed input pulse, because there is more energy in the pulse due to the filling and discharging periods of the pulse compressor. Before the klystron was replaced, the breakdown rate with the pulse compressor was $(1.9 \pm 0.3) \times 10^{-6} \mathrm{bpp}$ for $215 \mathrm{~ns}$ pulses of $20.5 \mathrm{MW}$. After the klystron was replaced, 8 million pulses later, with the pulse compressor detuned the breakdown rate was $(1.3 \pm 0.2) \times 10^{-6} \mathrm{bpp}$ for $200 \mathrm{~ns}$ pulses of $20.5 \mathrm{MW}$. With more pulsing, the breakdown rate continued to fall, so the cavity was still conditioning. Consequently, it is not clear if the compressed pulse is more likely to cause breakdown than an uncompressed pulse.

Over a period of months as logged in Fig. 6, the conditioning algorithm increased the power from $25 \mathrm{MW}$ and passed $45 \mathrm{MW}$ at 257 million pulses. The power was increased to $46 \mathrm{MW}$ for a short period at the 262 million pulse mark, but the operation was unstable with the BDR increasing toward $10^{-4} \mathrm{bpp}$ (not visible in Fig. 6). The power was lowered to $40 \mathrm{MW}$ to stop the breakdown clustering from damaging the structure, and control was handed back to the conditioning algorithm with a BDR set point of $3 \times 10^{-5} \mathrm{bpp}$ and a target power of $46 \mathrm{MW}$. Although the target was reached, the BDR oscillated above and below the set point, in turn causing the algorithm to ramp and decrease the power level. The $46 \mathrm{MW}$ power level that was reached appeared to be a hard limit, and it was believed that the structure was now approaching its fully conditioned state. With respect to Fig. 6, it is interesting that all the structures condition in roughly the same number of pulses. Figure 7 plots conditioning history as a function of the peak surface electric field and peak surface modified Poynting vector with comparison to accelerating structures. Conditioning scales identically for accelerating and dipole- 

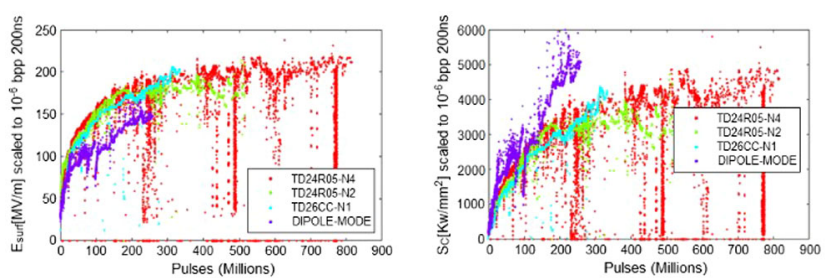

FIG. 7. Conditioning history of the dipole-mode cavity versus high-gradient monopole structures tested at CERN. Accelerating structure data are from Ref. [14].

mode structures when plotted versus both electric field and modified Poynting vector, although the conditioned fields are lower in electric field and higher in modified Poynting vector. In both cases, we compare with accelerating structure data from Ref. [14].

\section{STRUCTURE TEST}

After conditioning to a level consistent with its likely ultimate performance, it was then possible to get accurate measurements on the BDR dependence on power and pulse duration, as the structure will further condition slowly enough that different measurements can reliably be considered to the same level of conditioning. It was decided to first verify the BDR dependency on the pulse width at a fixed power level of $43 \mathrm{MW}$, where the structure could run in a stable state with $200 \mathrm{~ns}$ pulses while providing a sufficiently high breakdown rate that adequate statistics could be gathered within a few days for each different pulse width. The first point to be taken was the nominal $200 \mathrm{~ns}$ flattop point, followed by a $150 \mathrm{~ns}$ run. The pulse width was then briefly increased to $250 \mathrm{~ns}$, where the breakdown rate increased considerably. This pulse width was held only for 570000 pulses to reduce the chance of damage to the structure. Finally, the pulse width was lowered to 120 ns until the end of the run. A power law fit has been applied to the data, shown in Fig. 8(a), and has an exponent of $3.6 \pm 0.3$. This agrees to within $28 \%$ of the value reported in the literature. During the $250 \mathrm{~ns}$ run, the BDR peaked at $8 \times 10^{-4} \mathrm{bpp}$. Such a high concentration of breakdown events caused the structure's performance to deteriorate. However, the data point at $120 \mathrm{~ns}$ full width half maximum (FWHM) has a BDR higher than expected, because it was taken after the $250 \mathrm{~ns}$ point.

After measuring the BDR dependence on pulse length, the BDR dependence on power, utilizing a flattop pulse duration of $120 \mathrm{~ns}$, was determined. Normally, a structure is tested by starting at the highest power and then sequentially lowering the power with each point to ensure the structure does not degrade due to damage during the measurement. Here, the structure was initially run at the highest power of $52 \mathrm{MW}$, but, as an alternative procedure, the power was first stepped back to $45 \mathrm{MW}$, then stepped to $48 \mathrm{MW}$, and finally $50 \mathrm{MW}$. The $43 \mathrm{MW}$ BDR data point taken while
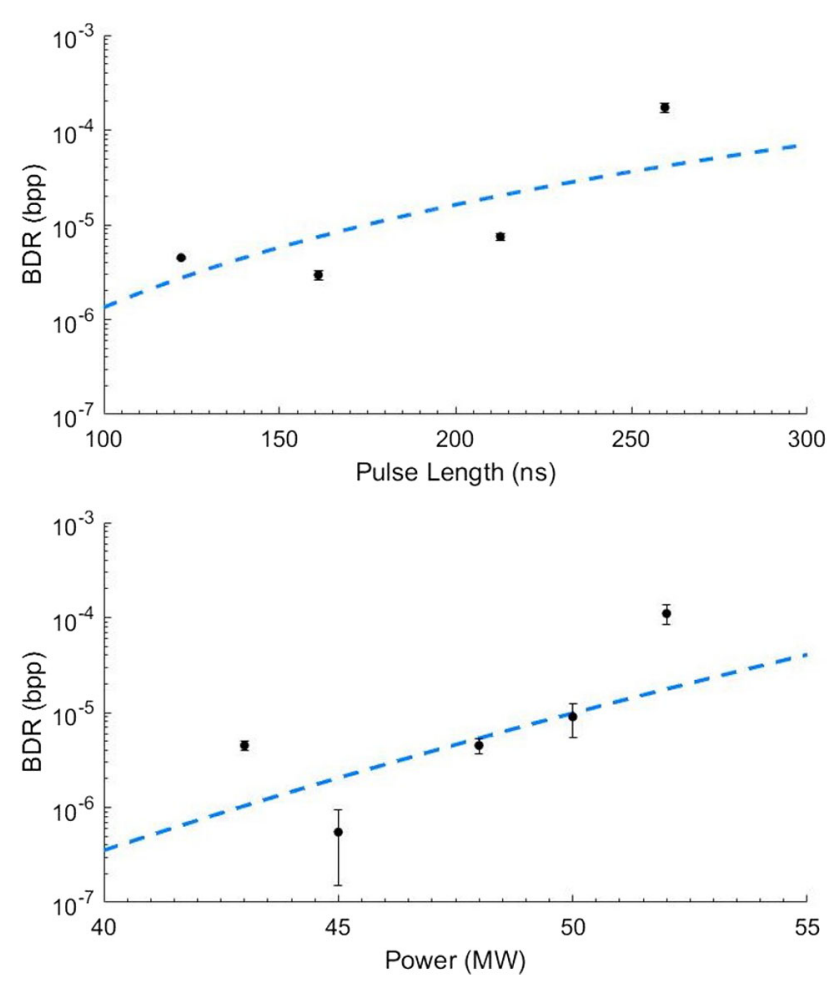

FIG. 8. Breakdown rate versus (a) pulse length at an input power of $43 \mathrm{MW}$ and (b) input power at a pulse length of $120 \mathrm{~ns}$.

measuring the dependence on pulse duration was included in the plots; however, it may correspond to a slightly lower level of conditioning with respect to the $45 \mathrm{MW}$ data point due to the sequence of measurements. Data taken at power levels of 43, 45, 48, 50, and $52 \mathrm{MW}$ points are plotted in Fig. 8(b) to show the relationship between power and BDR. A power law has been applied to the data with the best fit occurring with an exponent of $14.9 \pm 4.9$. This is consistent with the value reported in the literature of 15 [22]; however, the fit has a large $\chi^{2}$ value of 143 , indicating that there are some problems due to the limited number of points and the large uncertainty of the low BDR values of power.

As previously stated, the difference in time between the falling edge of the truncated transmitted signal and the rising edge of the reflection are used to localize the breakdowns to the cell in which they occurred within the structure. As the breakdown events typically occur at the regions of peak field situated on the iris, the difference in phase between the incident and reflected signals during breakdown tends to occur in multiples of the $120^{\circ}$ cell advance, and, hence, the phase information was used to reduce the uncertainty during localization. The distribution of breakdowns was regularly examined throughout testing to monitor for the emergence of "hot" cells with a BDR significantly higher than those surrounding it, which could be indicative of a defect or contamination, and indeed to monitor the conditioning progress of the structure 


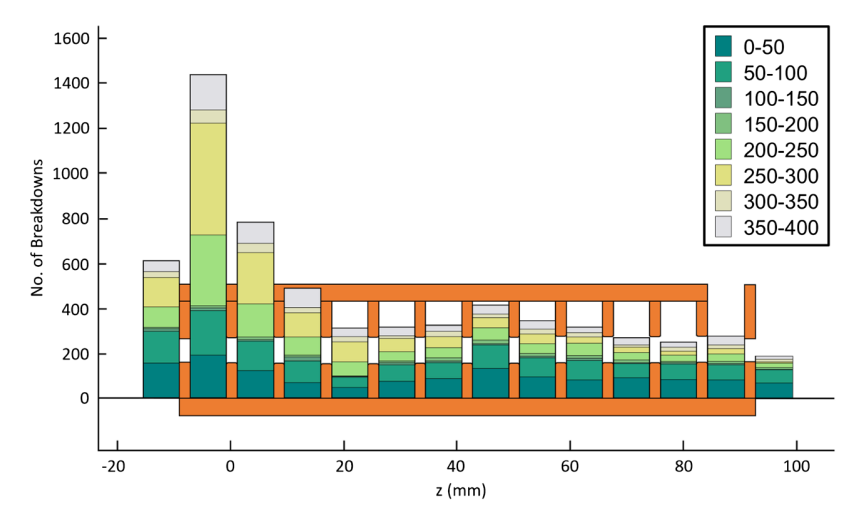

FIG. 9. Number of breakdowns in each cell for various windows of cumulative pulses.

as a whole. A total of 6351 breakdowns were logged as having occurred within the structure, and the results of the localization for each 50 million pulse window of testing are shown in Fig. 9. As the cavity has constant impedance, the field is highest in the first few cells; hence, one expects a higher number of breakdowns in those cells as seen. While the difference in field is less than $10 \%$, the breakdown rate should scale as $E^{30}$; hence, that would equate to a BDR that is 17.5 times higher in the first cell compared to the last. Other factors include the field asymmetry due to the coupler and the high magnetic fields on the coupler.

Of the breakdown events localized, 612 were found to have occurred in the waveguide immediately prior to the input coupler and 191 between the structure output coupler and the load. A review of randomly sampled waveforms in this set showed that of the events lying outside the structure approximately $20 \%$ were found to be due to errors in the identification of breakdown time from the rising and falling edges. Typically, the cause of such cases is due to the presence of oscillatory behavior in the waveform immediately prior to the breakdown as opposed to a characteristic sharp truncation. This is thought to be caused by energy deposition in the emission site prior to plasma formation; however, the exact explanation of the physical phenomena responsible for this behavior is beyond the scope of this paper.

The peak surface fields at operating power for the CLIC dipole-mode "crab" cavity, a CLIC T24 accelerating cavity, and the LCLS deflector are shown in Table I. Also shown is the CLIC crab cavity at $40 \mathrm{MW}$, which is equivalent to roughly a BDR of $10^{-6}$ similar to the T24 at operating power. The designed operating power of the CLIC crab cavity is likely too conservative, and higher fields can be sustained. At the maximum power of $40 \mathrm{MW}$, the CLIC crab cavity has a peak electric field which is $42 \%$ lower than the T24, a peak magnetic field which is $19 \%$ higher, and an $S_{c}$ which is $38 \%$ higher (with the square of $S_{c}$ being $21 \%$ higher). This suggests that it is the magnetic field or the square of $S_{c}$ which is the better predictor of structure performance or that dipole cavities cannot handle as high an electric field as accelerating cavities. The LCLS deflector has the same peak magnetic field as a T24 structure, while SLAC reports very few breakdowns at this field level [10]. The breakdown rate versus peak electric field and the square of $S_{c}$ are shown in Fig. 10 for various structures, showing that the dipole-mode cavity has the lowest $E_{\text {peak }}$ and second-highest $S_{c}$ of all structures at constant BDR. We compare the crab cavity to other

TABLE I. Comparison of operating fields for the crab cavity and other accelerating and dipole-mode cavities compared to the expected maximum operating gradient of the dipole-mode cavity.

\begin{tabular}{|c|c|c|c|c|}
\hline Property & $\begin{array}{l}\text { CLIC T24 } \\
\text { (unloaded) }\end{array}$ & LCLS deflector & $\begin{array}{c}\text { CLIC Crab } \\
\text { (undamped) operation }\end{array}$ & $\begin{array}{c}\text { CLIC Crab (undamped) } \\
\text { BDR } \sim 10^{-6}\end{array}$ \\
\hline Input power & $37.2 \mathrm{MW}$ & $20 \mathrm{MW}$ & $13.35 \mathrm{MW}$ & $40 \mathrm{MW}$ \\
\hline Transverse kick & $\ldots$ & $24 \mathrm{MV}$ & $2.55 \mathrm{MV}$ & $4.41 \mathrm{MV}$ \\
\hline Peak surf. E field & $219 \mathrm{MV} / \mathrm{m}$ & $115 \mathrm{MV} / \mathrm{m}$ & $88.8 \mathrm{MV} / \mathrm{m}$ & $154 \mathrm{MV} / \mathrm{m}$ \\
\hline Peak surf. $\mathrm{H}$ field & $410 \mathrm{kA} / \mathrm{m}$ & $405 \mathrm{kA} / \mathrm{m}$ & $292 \mathrm{kA} / \mathrm{m}$ & $505 \mathrm{kA} / \mathrm{m}$ \\
\hline Peak $S_{c}$ & $3.4 \mathrm{MW} / \mathrm{mm}^{2}$ & $\ldots$ & $1.83 \mathrm{MW} / \mathrm{mm}^{2}$ & $5.48 \mathrm{MW} / \mathrm{mm}^{2}$ \\
\hline dT $200 \mathrm{~ns}$ & $8 \mathrm{~K}$ & $33 \mathrm{~K}$ & $17 \mathrm{~K}$ & $51 \mathrm{~K}$ \\
\hline Group velocity & $1.8 \%-0.9 \% \mathrm{c}$ & $-3.2 \% \mathrm{c}$ & $-2.9 \% \mathrm{c}$ & $-2.9 \% \mathrm{c}$ \\
\hline No. cells & 24 & 117 & 12 & 12 \\
\hline Property & T18 (unloaded) & TD18 (unloaded) & TD24 (unloaded) & TD26 (unloaded) \\
\hline Input power & $53 \mathrm{MW}$ & $56.8 \mathrm{MW}$ & $40.5 \mathrm{MW}$ & $43 \mathrm{MW}$ \\
\hline Transverse kick & $\ldots$ & $\ldots$ & $\ldots$ & $\ldots$ \\
\hline Peak surf. E field & $232 \mathrm{MV} / \mathrm{m}$ & $234 \mathrm{MV} / \mathrm{m}$ & $196 \mathrm{MV} / \mathrm{m}$ & $199 \mathrm{MV} / \mathrm{m}$ \\
\hline Peak surf. $\mathrm{H}$ field & $301 \mathrm{kA} / \mathrm{m}$ & $600 \mathrm{kA} / \mathrm{m}$ & 391 kA/m & 399 kA/m \\
\hline Peak $S_{c}$ & $4.4 \mathrm{MW} / \mathrm{mm}^{2}$ & $4.5 \mathrm{MW} / \mathrm{mm}^{2}$ & $3.7 \mathrm{MW} / \mathrm{mm}^{2}$ & $3.8 \mathrm{MW} / \mathrm{mm}^{2}$ \\
\hline dT $200 \mathrm{~ns}$ & $18 \mathrm{~K}$ & $70 \mathrm{~K}$ & $30 \mathrm{~K}$ & $31 \mathrm{~K}$ \\
\hline Group velocity & $1.7 \%-1.03 \% \mathrm{c}$ & $2.23 \%-0.87 \% \mathrm{c}$ & $1.65 \%-0.83 \% \mathrm{c}$ & $1.65 \%-0.83 \% \mathrm{c}$ \\
\hline No. cells & 18 & 18 & 24 & 26 \\
\hline
\end{tabular}



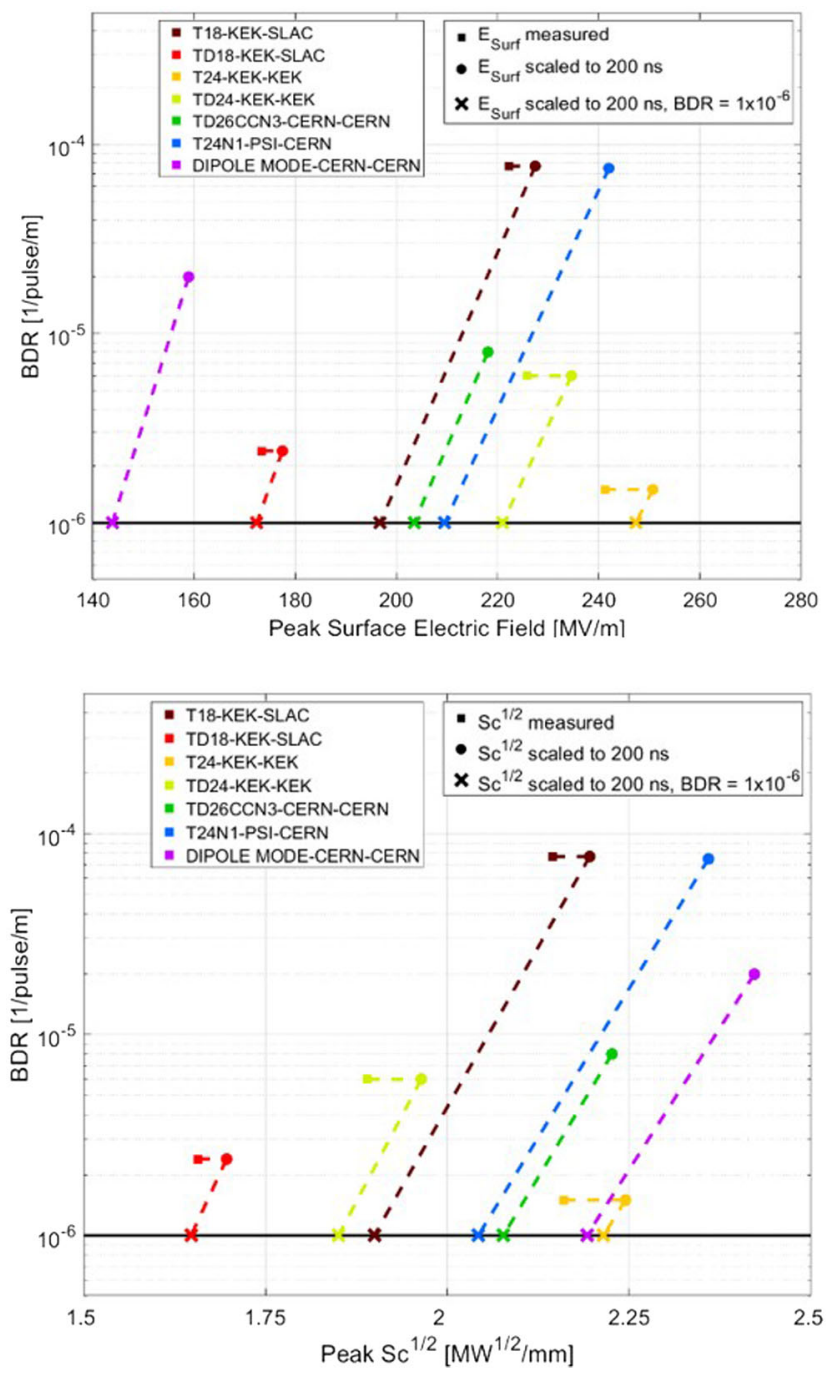

FIG. 10. The breakdown rate versus peak electric field and the square of $S_{c}$ for various structures.

structures at a BDR of $1 \times 10^{-6} \mathrm{bpp}$. Scaled to a breakdown rate of $1 \times 10^{-6}$, the cavity was found to operate at a peak electric field of $143.8 \mathrm{MV} / \mathrm{m}$ and a peak modified Poynting vector $S_{c}$ of $2.192 \mathrm{MW} / \mathrm{mm}^{2}$. The peak electric field is much lower than for accelerating structures, while the modified Poynting vector is moderately higher.

\section{POSTMORTEM AND ELECTRON MICROSCOPY}

After completion of the high-gradient testing, the cavity was examined by cutting the structure into parts to allow detailed examination of the modification of the cavity surfaces caused by the breakdowns. Wire electrical discharge machining was used for the cutting; however, some damage was caused by contamination in the electrolyte. Fortunately, this damage appeared as small spots and comet tails, thus very different to breakdown damage craters, like

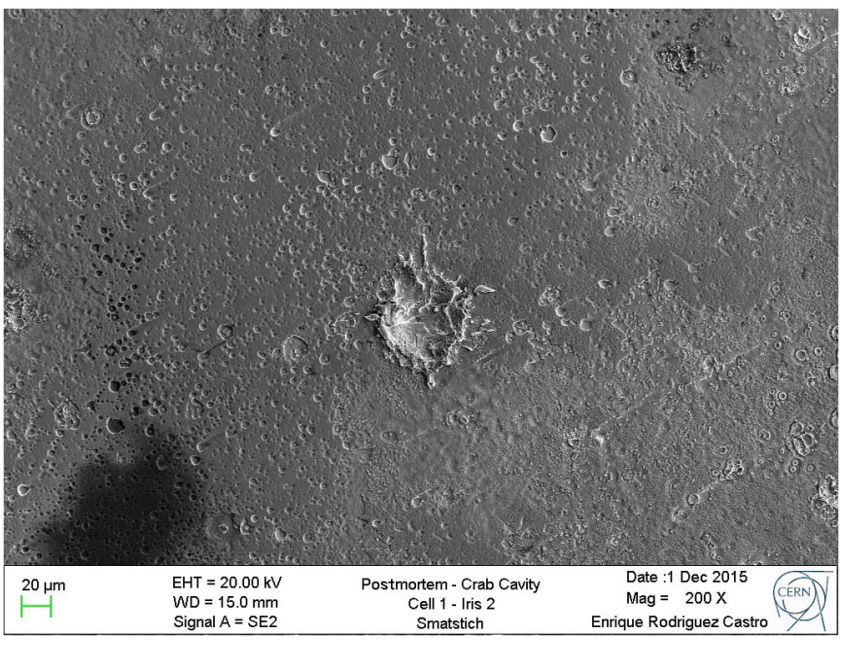

FIG. 11. SEM image of an isolated breakdown crater on the CLIC dipole-mode cavity.

the one in the center of Fig. 11, so the two effects could be separated.

One of the most interesting aspects of a dipole cavity test is that the peak electric and magnetic fields are located in different regions of the cavity, allowing the role of each component to be studied by observing where the breakdown craters occur. The location of the craters in each cell were recorded using an electron microscope. The breakdown crater position is plotted over the electric field, magnetic field, and modified Poynting vector in Fig. 12 for the input cell. As can be seen, the crater location correlates strongly with the peak electric field location, with most breakdowns occurring at the region of highest field opposite the coupler. For a modified Poynting vector, the breakdowns do fall mostly in the moderately high regions but very little at the peak location on the same side as the coupler.

In addition to breakdown craters, it is also possible to look for fatigue caused by pulsed rf heating in the high magnetic field regions. In an accelerating structure, the maximum magnetic field is on the outer cavity wall. In dipole cavities, the peak magnetic field region is at the iris in the vertical plane only, and very few breakdown craters are found there. Interestingly, there were no observable signs of fatigue at the peak magnetic field location despite the peak magnetic field being significantly higher than the fields normally achieved in accelerating cavities, as shown in Fig. 13.

To further study the field dependence of breakdown, the $x$ and $y$ positions of each breakdown crater were logged and converted into angles around the cavity center, with respect to the vertical axis. The breakdown crater locations versus angle is shown in Fig. 14 for the second iris. In cell 1, the electric field is asymmetric due to the effect of the coupler, hence explaining why there are more breakdowns at $90^{\circ}$ than at $270^{\circ}$. 

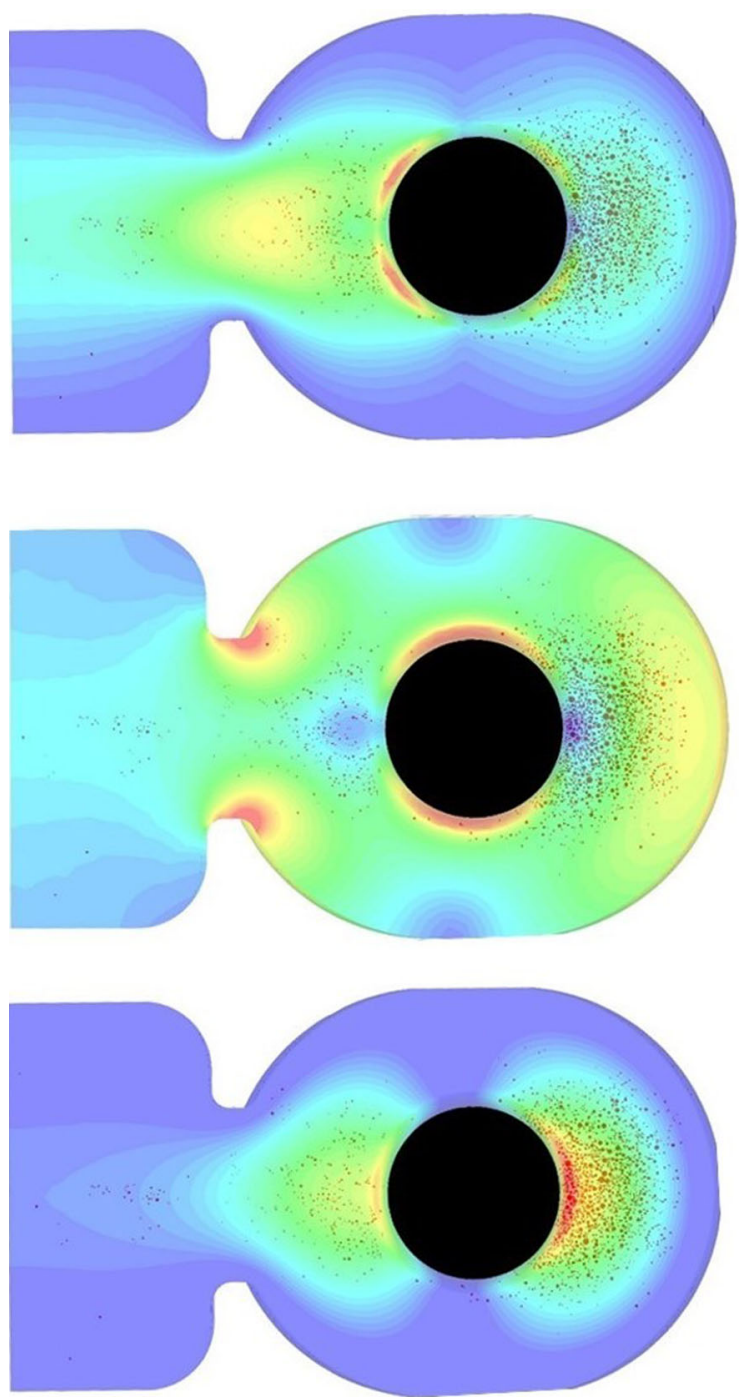

FIG. 12. Breakdown crater locations in the input cell plotted over (a) modified Poynting vector $S_{c}$, (b) magnetic field, and (c) electric field distributions from HFSS.

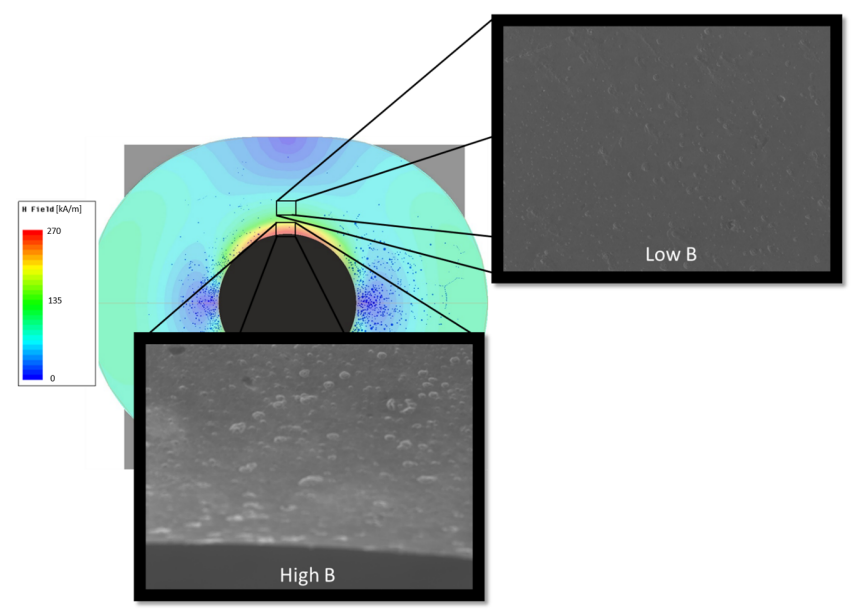

FIG. 13. Comparing surface SEM images for high and low magnetic field locations.

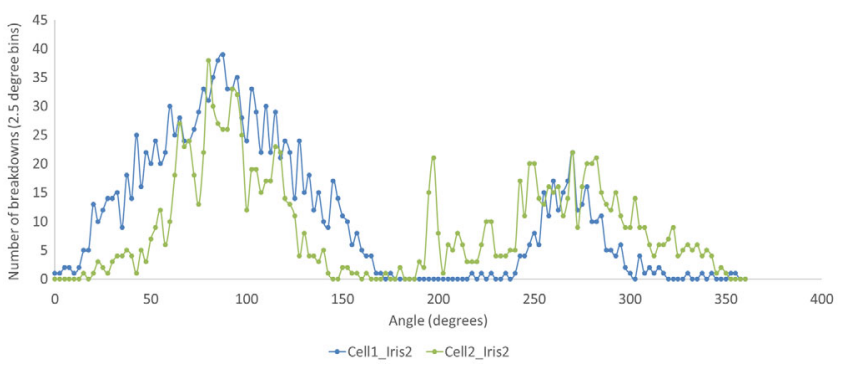

FIG. 14. The breakdown crater locations versus angle for the second iris, showing the cavity 1 side in blue and cavity 2 side in green.

In cell 2, there is a cluster of breakdowns at $195^{\circ}$ in a $\mathrm{Y}$-shaped pattern, shown in Fig. 15. It is suspected that these follow a grain boundary. If the $\mathrm{Y}$-shaped cluster is neglected, the angular distribution of pits is a smoother distribution and can be fitted to a sinusoidal function. In a conditioned structure, the breakdown rate for the whole structure varies as a large power of the electric field; however, this scaling would not be expected to be
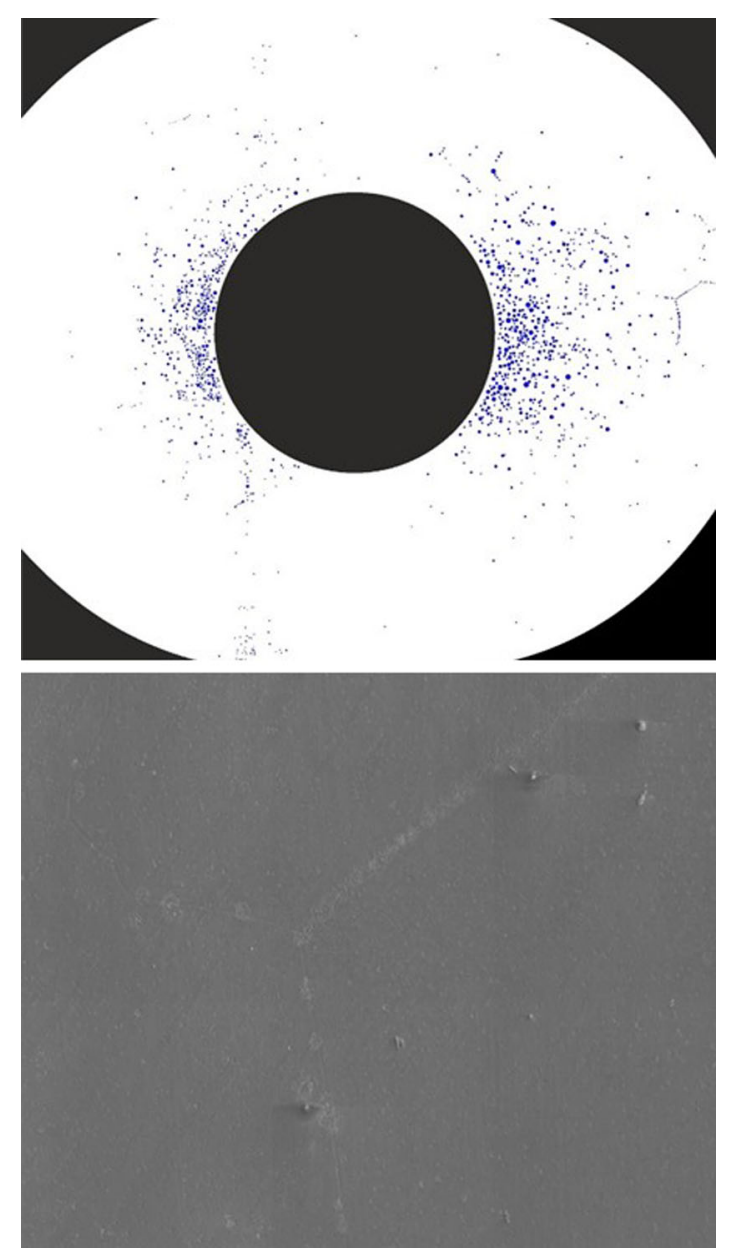

FIG. 15. (a) Breakdown location in cell 2 iris 2, and (b) SEM image of the $\mathrm{Y}$-shaped breakdown cluster. 


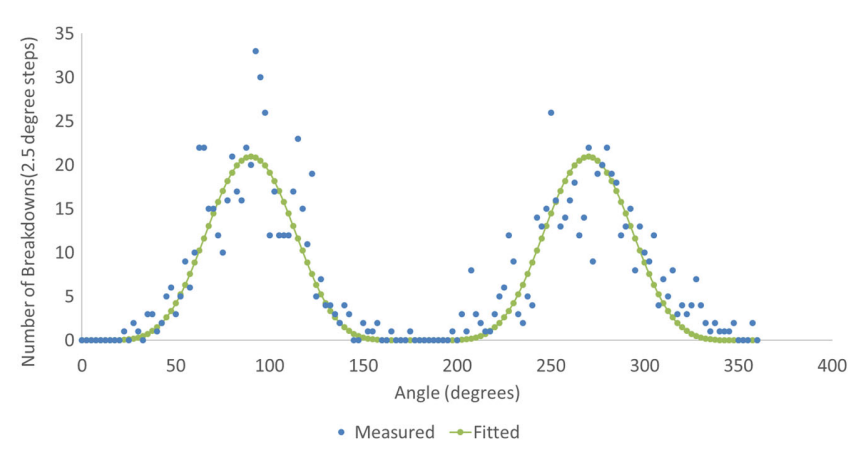

FIG. 16. Breakdown crater location angular distribution in cell 2 iris 2 fitted against $\sin ^{6}(\phi)$.

maintained on a local scale, as different parts of the structure are conditioned to different electric fields due to the modal field distribution. For example, if the electric field at $90^{\circ}$ is conditioned to $100 \mathrm{MV} / \mathrm{m}$, the surface at $30^{\circ}$ will be conditioned only to $50 \mathrm{MV} / \mathrm{m}$. The fact that there is still an angular dependence on breakdown location suggests that a structure conditioned to a higher gradient running at that gradient will still have a higher breakdown rate than a structure conditioned to a lower gradient running at that lower gradient.

The breakdown density variation was fitted to fit to a function of form $\mathrm{BD}=A * \sin ^{m}(\phi)^{*}\left[1+B^{*} \operatorname{Cos}^{n}(\phi)\right]$, where $\phi$ is the angle with respect to the vertical axis where the magnetic field is highest, A and B are scaling constants, and $\mathrm{m}$ and $\mathrm{n}$ are the power constants. In dipole-mode cavities, the electric field varies as $\sin (\phi)$, and the magnetic field varies with $\cos (\phi)$; hence, this fit can tell us something about field dependence of breakdown on a local scale after conditioning. It was found that if $B=0$, i.e., fitting to only an electric field, we get a good fit to $m=6$ (shown in Fig. 16). If the magnetic field dependence is considered, there is a reasonable fit to $m=6, n=4$ where $\mathrm{B}$ is 9 times larger than A. Taking this into account, the breakdown rate should be proportional to the integral of the electric field to the power six integrated over the cavity surface. It should be noted that this scaling of the breakdown rate applies only to the electric field the local surface has been conditioned to and not the applied electric field, which scales more sharply. This is due to the fact that low field regions of the cavity will not be conditioned to as high a field as a high field region, and for a given input power the local electric field will vary over the surface.

\section{CONCLUSION}

It has been shown that dipole-mode cavities the field levels reached for a given BDR are lower for an electric field and slightly higher for a modified Poynting vector and magnetic field, suggesting that these may be better indicators of breakdown performance. From the postmortem examination, the breakdown crater locations were found to correlate strongly with the peak electric field, and there were neither breakdown craters nor fatigue damage in the high magnetic field regions. Such a distinction is not possible in accelerating structures, as the peak fields both occur in the same location; hence, this result gives an interesting insight into the role of both fields in breakdown. Thus, these test results provide important input for improving the quantities which give high-gradient limits and, consequently, the high-gradient structure design process. The field levels at a breakdown rate of $10^{-6}$ are $154 \mathrm{MV} / \mathrm{m}$ and $505 \mathrm{kA} / \mathrm{m}$ for an electric and a magnetic field, respectively. It is also found that dipole-mode structures condition at the same rate in terms of number of pulses as accelerating structures.

These results suggest that dipole-mode cavities could operate at almost twice the gradients currently being used if a lower group velocity or higher power amplifier were used, or a larger aperture could be used to reduce wakefields.

Additionally, we analyze the angular dependence of the breakdown crater locations, due to the strong angular dependence of the electric and magnetic fields with the angle in dipole-mode cavities. We find the breakdown density is proportional to a power law fit to the electric field with an exponent of six, suggesting that for structures operating at the field they are conditioned to have a breakdown rate proportional to the conditioning electric field to the power of six.

\section{ACKNOWLEDGMENTS}

This work was funded by STFC via the Cockcroft institute grant (No. ST/P002056/1). The authors thank Valery Dolgashev for advice in the structure design and all the staff and students involved in the setup of the XBox test stands at CERN.

[1] G. Burt, Transverse deflecting cavities, arXiv:1201.2600.

[2] D. Xiang and Y. Ding, Longitudinal-to-transverse mapping for femtosecond electron bunch length measurement, Phys. Rev. Accel. Beams 13, 094001 (2010).

[3] M. Cornacchia and P. Emma, Transverse to longitudinal emittance exchange, Phys. Rev. Accel. Beams 5, 084001 (2002).

[4] M. Borland, Simulation and analysis of using deflecting cavities to produce short X-ray pulses with the Advanced Photon Source, Phys. Rev. Accel. Beams 8, 074001 (2005).

[5] D. A. Edwards, An rf separated kaon beam from the main injector: Superconducting aspects, Report No. FERMILAB-TM-2060, Fermi National Accelerator Laboratory, Batavia, IL, 1998.

[6] R. B. Palmer, Energy scaling, crab crossing and the pair problem, Report No. SLAC-PUB-4707, Stanford Linear Accelerator Center, 1988. 
[7] A. Grudiev, S. Calatroni, and W. Wuensch, New local field quantity describing the high gradient limit of accelerating structures, Phys. Rev. Accel. Beams 12, 102001 (2009).

[8] K. Nordlund and F. Djurabekova, Defect model for the dependence of breakdown rate on external electric fields, Phys. Rev. Accel. Beams 15, 071002 (2012).

[9] D. P. Pritzkau and R. H. Siemann, Experimental study of rf pulsed heating on oxygen free electronic copper, Phys. Rev. Accel. Beams 5, 112002 (2002).

[10] V. A. Dolgashev, G. Bowden, Y. Ding, P. Emma, P. Krejcik, J. Lewandowski, C. Limborg, M. Litos, J. Wang, and D. Xiang, Design and application of multimegawatt X-band deflectors for femtosecond electron beam diagnostic, Phys. Rev. Accel. Beams 17, 102801 (2014).

[11] G. Burt, P. McIntosh, R. Wegner, A. Solodko, A. Grudiev, B. Woolley, W. Wuensch et al., Prototype development of the CLIC crab cavities, in Proceedings of Linac14 (Jacow, Geneva, 2014), THPP013.

[12] P. K. Ambattu, G. Burt, V. F. Khan, R. M. Jones, A. Dexter, and V. Dolgashev, Analysis and control of wakefields in X-band crab cavities for Compact Linear Collider, Nucl. Instrum. Methods Phys. Res., Sect. A 657, 27 (2011).

[13] A. C. Dexter, G. Burt, P. K. Ambattu, V. Dolgashev, and R. Jones, CLIC crab cavity design optimisation for maximum luminosity, Nucl. Instrum. Methods Phys. Res., Sect. A 657, 45 (2011).
[14] A. Degiovanni, W. Wuensch, and J. G. Navarro, Comparison of the conditioning of high gradient accelerating structures, Phys. Rev. Accel. Beams 19, 032001 (2016).

[15] VDL, https://www.vdlsystems.nl/nl.

[16] Bodycote, https://www.bodycote.com/.

[17] R. Wegner, G. Burt, B. Woolley, and W. Wuensch, Bead-pull measurement method, and tuning of a prototype CLIC crab cavity, in Proceedings of Linac14 (Jacow, Geneva, 2014), MOPP035.

[18] J. G. Navarro, High-gradient structure performances: Ongoing tests and summary, in Proceedings of the CLIC Workshop, 2016.

[19] B. Woolley, I. Syratchev, and A. Dexter, Control and performance improvements of a pulse compressor in use for testing accelerating structures at high power, Phys. Rev. Accel. Beams 20, 101001 (2017).

[20] M. Jacewicz, Spectrometers for rf breakdown studies for CLIC, Nucl. Instrum. Methods Phys. Res., Sect. A 828, 63 (2016).

[21] A. Degiovanni, B. Woolley, W. Wuensch, W. Farabolini, S. Döbert, I. Syratchev, J. G. Navarro, and J. Tagg, Diagnostics, and analysis techniques for high power X-band accelerating structures, in Proceedings of Linacl4 (Jacow, Geneva, 2014), TUPP029.

[22] A. Degiovanni, W. Wuensch, and J. G. Navarro, Comparison of the conditioning of high gradient accelerating structures, Phys. Rev. Accel. Beams 19, 032001 (2016). 